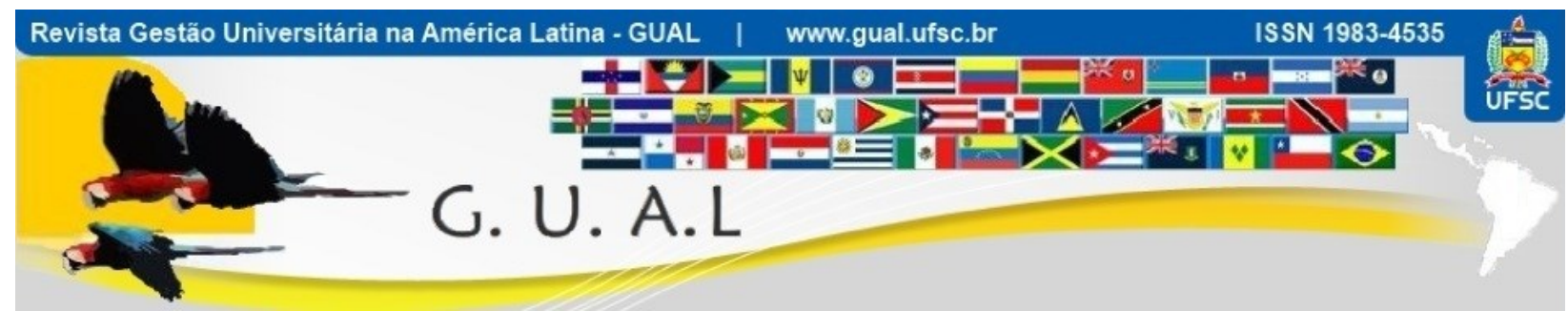

DOI: http://dx.doi.org/10.5007/1983-4535.2013v6n4p23

\title{
PROPENSÃO À ADOÇÃO DE NOVAS TECNOLOGIAS POR PROFESSORES NA PRODUÇÃO DE CONTEÚDOS EDUCACIONAIS: UM ESTUDO COM BASE NA TEORIA DA DIFUSÃO DE INOVAÇÕES
}

\author{
PROMPTNESS TO NEW TECHNOLOGY ADOPTION BY LECTURES IN THEIR \\ EDUCATIONAL MATERIAL PRODUCTION ACTIVITIES: A STUDY BASED ON \\ THE INNOVATION DIFFUSION THEORY
}

Telma Regina da Costa Guimarães Barbosa, Doutora Universidade Federal de Viçosa - UFV telma@ufv.br

Maria de Lourdes de Carvalho, Mestre Universidade Federal de Viçosa - UFV lurdinha@ufv.br

Silvane Guimarães Silva Gomes, Doutora Universidade Federal de Viçosa - UFV silvane@ufv.br

João Batista Mota, Graduação Universidade Federal de Viçosa - UFV joaomota@ufv.br

Recebido em 29/novembro/2013

Aprovado em 29/novembro/2013

Sistema de Avaliação: Double Blind Review

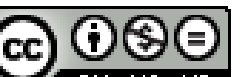

Esta obra está sob uma Licença Creative Commons Atribuição-Uso. 


\title{
RESUMO
}

Objetiva caracterizar os diferentes graus de aceitação das nTICs pelos professores participantes de um projeto de novas tecnologias aplicadas ao ensino, bem como identificar os fatores influenciadores da aceitação com base em algumas atributos do processo de adoção de inovação. É um estudo exploratório, realizado após a conclusão de um projeto de nTICs, onde se define a propensão para aceitação das novas tecnologias com base em três constructos: conclusão, experimentação e intenção de ampliar. As variáveis independentes foram retiradas do modelo de Rogers (2003) e de outros autores que ampliaram esse modelo. Dados foram analisados por meio de estatísticas descritivas, tabelas de contingência, e teste do Chi-Square de Pearson. Conclui-se que apenas os constructos experimentação e intenção de ampliar puderam ser explicados pelas variáveis independentes e que as principais explicações encontram-se na categoria de atributos pessoais. Os atributos de vantagens percebidas e influências interpessoais respondem apenas razoavelmente pela propensão a adoção.

Palavras-chave: Adoção de novas tecnologias de informação e comunicação. Prontidão. Características pessoais. Atributos de percepção. Influências interpessoais pessoais.

\begin{abstract}
The aim is to understand the differing levels of new technology of information and communication adoption amongst lectures who took part of a project of applying new technologies to teaching and learning practices. It also aims at identifying the major factors influencing the differing levels of technology adoption. Technology adoption promptness was defined by means of three constructors: work conclusion rate, work experimentation and intention to apply to teaching activities. Independent variables were based on the Innovation Diffusion Theory (Rogers, 2003) and others. Descriptive statistics, contingency tables and Pearson chi-square applied. The results suggested that the constructors work experimentation and intention to apply to teaching activities were more adequately explained by the independent variables. Amongst those, personal attributes are the most relevant influencing factors, followed by perceived advantages and interpersonal influences.
\end{abstract}

Keywords: New Technology of information and communication adoption. Promptness. Personal characteristics. Perceptions atributes. Interpersonal influences. 


\section{INTRODUÇÃO}

Organizações em geral enfrentam constantes mudanças tecnológicas e, para fazer frente a essas mudanças, a inovação tem sido enfatizada. Nesse contexto, pesquisadores de vários campos tem-se dedicado ao estudo da adoção de inovações na tentativa de se prever os fatores influenciadores da adoção e difusão de inovações.

As novas tecnologias de informação e comunicação (nTICs) como recursos e mídias voltadas para o processo educacional tornam-se cada vez mais relevantes por representarem novas possibilidades para o ensino-aprendizagem. Essas tecnologias evidenciam a possibilidade de interação, de comunicação, de acesso à informação e convertem-se em um meio interativo e ativo no processo educacional (TORI, 2002; PUNIE, 2002).

Nesse sentido, as instituições de ensino têm aproveitado as possibilidades oferecidas pelas nTICs e visualizam uma opção viável para os seus programas de ensino, em todos os níveis e modalidades. Assim, o uso de nTICs tornou-se claramente um dos focos atuais no sistema educativo de todo o país. Com elas, a reorganização do ensino influencia diretamente a prática docente a partir da seleção dos meios mais apropriados para determinada situação de ensino-aprendizagem, considerando os objetivos pedagógicos e didáticos previamente definidos, bem como as características dos estudantes e acessibilidade aos meios.

$\mathrm{Na}$ prática, argumenta-se que a utilização das nTICs permite ao professor mais liberdade na apresentação da disciplina e, como consequência, melhor adequação dos conteúdos para atender aos diferentes estilos de aprendizagem e perfis dos estudantes.

Neste contexto a Coordenação de Aperfeiçoamento de Pessoal de Nível Superior CAPES, em parceria com a Secretaria de Educação Superior (SESu) e a Secretaria de Educação a Distância (SEED), do Ministério da Educação - MEC publicou o Edital Fomento ao uso das Tecnologias de Comunicação e Informação nos cursos de Graduação (edital $n^{\circ}$ 015/2010/CAPES/DED), com o objetivo de "favorecer a institucionalização de métodos e práticas de ensino-aprendizagem inovadores que, baseados no uso das tecnologias da comunicação e da informação, promovam a integração e a convergência entre as modalidades de educação presencial e a distância nas IES integrantes do sistema UAB; e, ainda, criar e/ou incrementar uma cultura acadêmica que tenha no uso de recursos tecnológicos avançados um instrumento útil para a otimização da gestão universitária."

A Universidade Federal de Viçosa (UFV), por meio da Coordenadoria de Educação Aberta e a Distância (CEAD) é uma das instituições participantes desse projeto. Para tanto, 
foram priorizadas disciplinas que completam a matriz curricular de vários cursos e são oferecidas a um grande número de alunos em várias turmas. Desta forma, atinge-se um grande número de estudantes matriculados nos mais diferentes cursos das diversas áreas do saber.

O projeto envolveu a participação de 54 professores com seus tutores e estagiários os quais se responsabilizaram pela produção de material didático para as disciplinas selecionadas. O processo de elaboração do material didático teve início com oficinas onde participantes tomaram conhecimento dos diferentes formatos de material (apostilas interativas, áudio-aulas quadro-digital, captura de tela, vídeo-aulas, simulações e imagens animadas em flash e filmes de práticas em campo ou em laboratório) e de como produzi-los. A escolha do material a ser produzido e a elaboração do cronograma de trabalho foram realizadas pelos próprios participantes. Ao longo do processo foram realizadas reuniões e oficinas de orientação de modo individual para atender necessidades especificas de disciplinas ou de professores. Além disso, todo o processo foi acompanhado por uma equipe da CEAD não só para monitorar os resultados como também para incentivar a conclusão do projeto, numa dinâmica interativa e baseada na reflexão-ação-reflexão.

Ao final do projeto, embora se tenha obtido sucesso com a realização dos objetivos, a despeito dos esforços, o desempenho e a produtividade do grupo não foram uniformes. Muitos professores se mostraram entusiasmados e satisfeitos, porem as taxas de conclusão, de experimentação e de intenção de adoção e ampliação dos materiais variaram entre os participantes.

A partir deste resultado, o presente estudo pretende caracterizar os diferentes graus de aceitação das nTICs pelos professores participantes do projeto e identificar os fatores influenciadores da aceitação, com base em algumas características do processo de difusão de inovação

Estudar a motivação para a adoção de nTICs nesse contexto é importante, pois esse conhecimento auxilia na gestão da introdução das NTICs no ensino.

\section{FUNDAMENTAÇÃO TEÓRIA}

\subsection{ADOÇÃO DE TECNOLOGIA DA INFORMAÇÃO (TI) E FATORES} INFLUENCIADORES

Em um estudo sobre adoção de inovações em tecnologias de informação, Jeyaraj, Rottman e Lacity (2006) identificaram onze principais teorias e modelos teóricos preditores 
da adoção. Entre essas, a maior parte objetiva avaliar a adoção do ponto de vista do individuo e são as teorias denominadas Teoria da Ação Racionalizada, de Fishbein e Ajzen, (1975); Teoria da Difusão da Inovação (TDI), de Rogers (1983, 1985); Teoria Cognitiva Social, de Bandura (1986); Modelo de Aceitação de Tecnologia (TAM), de Davis (1989); Teoria do Comportamento Planejado (TPB), de Ajzen (1991); Características Percebidas da Inovação, de Moore e Benbasat (1991); Teoria Unificada de Aceitação e Uso de Tecnologia (UTAUT), de Venkatesh et al (2003) e Teoria Ator-rede, de Latour (2003).

A Teoria da Difusão da Inovação (TDI) e o Modelo de Aceitação de Tecnologia (TAM) são as mais frequentemente mencionadas. Nessas teorias, dois temas são relevantes: o processo e a taxa de adoção e os fatores influenciadores da adoção da inovação.

Rogers (2003), um dos principais autores de referência na área, e outros que compartilham suas idéias, tais como Cooper e Zmud (1989) e Huff e McNaughton (1991) caracterizam a adoção de TI como um processo. Rogers (2003), na teoria da Difusão de Inovação (DTI), define que a taxa de adoção é a velocidade relativa com que uma inovação é adotada por um grupo de indivíduos. Para o autor a adoção é um processo de tomada de decisão pelo individuo quanto ao uso da inovação como melhor curso de ação disponível. O processo ocorre em cinco estágios: conhecimento; persuasão; decisão; implementação e confirmação. Cooper e Zmud (1989) sugerem um processo de seis fases: iniciação, adoção, adaptação, aceitação, rotinização e infusão. Huff e McNaughton (1991) sugerem um processo de cinco etapas: conhecimento inicial da inovação, formação de uma atitude em relação à inovação, decisão de adotar/ou rejeitar, uso da inovação, reforço à decisão de adotar. Nesse sentido, a defesa é de que o usuário passa por estágios sucessivos de intenção em adotar, comprometimento, hábito até a utilização profunda e integrada da inovação. A adoção não é simplesmente uma aceitação da inovação, mas sim seu emprego e a exploração total de seu potencial. Segundo os estudiosos, uma lacuna é evidenciada ao se compararem as taxas de adoção e de uso de uma nova TI ao longo do tempo. A essa lacuna Fichman e Kemerer (1999) denominam lacuna de assimilação.

Vários fatores são apontados como influenciadores da adoção de inovações. As teorias da Difusão de Rogers (2003), conhecida por DTI e do Modelo de Aceitação Tecnológica (TAM) têm sido largamente utilizadas para explicar a adoção de TI tanto do ponto de vista organizacional quanto individual. A Teoria da Difusão defende que a adoção de inovações, incluindo as de TI, depende mais dos atributos percebidos da inovação do que de seus 
atributos técnicos. Os atributos percebidos são: vantagem relativa, compatibilidade, testabilidade e observabilidade, que exercem uma influência positiva na taxa de adoção, e complexidade, que exerce uma influência negativa (ROGERS, 2003). A esses cinco atributos Rogers acrescenta quatro fatores organizacionais: tipo de decisão, a natureza dos canais de comunicação, sistema social, e esforço do promotor da mudança.

Os atributos percebidos de Rogers têm sido considerados os atributos universais para estudos de adoção de novas tecnologias (PEREZ, 2006) e têm sido validados e ampliados por outros pesquisadores. De acordo com Santos (2007) esses fatores foram validados para o caso de inovações em TI por Moore e Benbasat (1991) que incluíram um conjunto de novos fatores, quais sejam, voluntariedade do uso da inovação, imagem ou status do usuário da inovação, facilidade de uso, demonstrabilidade dos resultados do uso da inovação. Vale ressaltar que Moore e Benbasat (1991) defendem que a percepção dos atributos por meio do uso da inovação exerce papel mais relevante como influenciador do processo de adoção do que as características em si. Ou seja, mais relevante do que "o grau com o qual uma inovação é percebida como sendo melhor que sua precursora" é o "grau com o qual o uso de uma inovação é percebido como melhor do que o uso de sua precursora".

Ainda nessa linha, Gatignon e Robertson (1991) também ampliaram os conceitos fundamentais da teoria da difusão de Rogers e propuseram a inclusão de estratégias de marketing para a inovação e atividades competitivas, entre as variáveis organizacionais, e as influencias interpessoais e características pessoais dos inovadores e adotantes, entre os fatores individuais.

O Modelo da Aceitação de Tecnologia (TAM) e suas evoluções buscam identificar os motivos que levam indivíduos (e organizações) a aceitarem ou rejeitarem a nova tecnologia. Para tal propõem uma análise do impacto de variáveis externas nas crenças, atitudes e intenções dos indivíduos em uma organização, mediado pelos dois fatores mais relevantes do modelo: utilidade percebida e facilidade percebida do uso (DAVIS, 1989, DAVIS, BAGOZZI e WARSHAW, 1989). Esses fatores têm o mesmo significado de vantagem relativa e facilidade de uso, respectivamente, do modelo de Rogers. Apesar dessa similaridade, argumenta-se que o modelo não aborda a introdução de TI (PEREZ, 2006). 


\section{METODOLOGIA}

Este estudo deriva-se da experiência vivenciada pela equipe da Coordenadoria de Educação Aberta e a Distância (CEAD) de uma Instituição Federal de Ensino Superior (IFES) com professores envolvidos na produção de conteúdos educacionais para cursos de graduação presenciais, de acordo com o Edital $n^{\circ}$ 015/2010/CAPES/SEED/MEC, denominado Projeto nTICs.

Para o desenvolvimento do estudo foi realizada uma pesquisa exploratória com o objetivo de levantar as principais características que classificam os professores em termos da sua propensão para aceitação das novas tecnologias. A coleta de dados se deu por meio de um questionário estruturado e foi realizada após a conclusão do projeto nTICs.

A população do estudo é composta pelos 54 (cinquenta e quatro) professores participantes do projeto nTICs. Vale ressaltar que a participação desses professores se deu a convite da Coordenadoria de Educação Aberta e a Distância - CEAD. Portanto, todos os participantes concordaram com os termos do projeto. Oito professores não responderam à solicitação desse estudo e, assim a amostra final compõe-se de 46 professores. Destes, 15,2\% fazem parte do Centro de Ciências Agrárias; 34,8\% fazem parte do Centro de Ciências Biológicas e da Saúde; 41,3\% são do Centro de Ciências Exatas e Tecnológicas e 8,7\% são do Centro de Ciências Humanas, Letras e Artes.

Partindo do suposto que quanto mais uma tecnologia é utilizada pelo individuo mais ela é adotada por ele, a propensão à adoção de novas tecnologias de informação e comunicação (nTICs) nesse estudo foi operacionalizada por meio de três constructos:

a) Status da produção de material (STS) - definido pelas categorias "completo" e "incom-pleto";

b) Grau de experimentação (EXP), dado pelas categorias - "nenhum ou não", "em parte" e "sim"; e

c) Grau de intenção de continuar/ampliar a utilização das nTICS na produção de materiais didáticos (INT) nas categorias - "não", "sim, só concluir" e "concluir e ampliar". 
As variáveis independentes compreendem as seguintes categorias:

1. Características pessoais baseadas em Pádua Júnior e Prado (2006), sendo:

- Experiência com produtos/assuntos relacionados às nTICs e experiência com produtos/ assuntos relacionados ou à EAD;

- Familiaridade com as nTICs e familiaridade com a EAD;

- Habilidade para executar tarefas relacionadas às nTICs e habilidade para executar tarefas relacionadas às $\mathrm{EaD}$;

- Conhecimento prévio de tarefas relacionado às nTICs e conhecimento prévio tarefas relacionado à $\mathrm{EaD}$; e

- Característica inovadora: grau de esforço que o adotante está disposto a realizar para adquirir uma inovação assim que ela é lançada.

Para todas as variáveis da categoria características pessoais espera-se uma associação positiva com as variáveis dependentes.

2. Características percebidas do modelo de Rogers (2003), sendo:

- Vantagens relativas das nTICs sobre métodos atuais na produção de conteúdo educacional;

- Compatibilidade das nTICs com os valores existentes no meio do adotante em relação à produção de conteúdo educacional;

- Experimentabilidade: facilidade no uso e funcionamento das nTICS bem como de dissipar incertezas a seu respeito; e

- Complexidade/dificuldade de compreensão e uso das nTICs na produção de conteúdo educacional.

Para as três primeiras variáveis da categoria características percebidas espera-se uma associação positiva com as variáveis dependentes e, para a última na sequencia, espera-se uma associação negativa.

3. Influências interpessoais relativas ao grupo de referência, adaptadas de Pádua Júnior e Prado (2006) e aqui agrupadas em:

- Informativas: procura informações com pessoas que trabalham na profissão; e pessoas que trabalham na profissão procuram informações com o adotante;

- Utilitárias: decisões do grupo são influenciadas pelas preferências e ações do respondente e decisão é influenciada pelas preferências e ações do grupo;

- De valor: uso das nTICs ajudará na promoção da imagem do respondente perante seus pares, ações do respondente influenciam na promoção de sua imagem; e ações do respondente influenciam a ação de seus pares. 
Para essas variáveis esperam-se também associações positivas da influencia do respondente em seu grupo.

Estas variáveis foram medidas por meio de uma escala de cinco pontos ( 1 - muito pouco/quase nenhum/ruim a 5 - Muito grande/ totalmente/muito bom).

Os dados foram analisados com base em estatísticas descritivas, tabela de contingência, e no teste do Chi-Square de Pearson, através do software de análise estatística SPSS (Statistic Package for Social Science), versão 11.5. A rejeição/adoção da hipótese nula (h0) de que não há associação entre as variáveis foi guiada pelo .Chi-Square tabulado de 9,488, para o nível de significância de 0,05 com grau de liberdade igual a 4 .

\section{ANÁLISE E RESULTADOS}

Classificando-se os respondentes pelo grau de propensão à adoção, tem-se que 71,7\% completaram o material e $28.3 \%$ não completaram (STS); $43.5 \%$ não experimentaram; 45,7\% experimentaram em parte e $10.9 \%$ experimentaram adequadamente o material desenvolvido (EXP) e, finalmente, $4.3 \%$ não tem interesse em concluir/ampliar; $45.5 \%$ tem certo interesse; $16,9 \%$ pretendem de fato concluir e $32,6 \%$ pretendem ampliar para outras disciplinas (INT).

O teste do Chi-Square revela que a variável supostamente dependente STS - Status da produção de material (incompleto/completo) não está associada a nenhuma das variáveis independentes em estudo, sendo todos os Chi-Square calculado menores que o Chi-Square tabelado definido para esse estudo. Vale aqui a menção de que nem mesmo os atributos relativos às influências interpessoais podem explicar a distinção nessa variável dependente. Poder-se-ia esperar que pessoas mais influentes entre seus pares tivessem comportamento diferenciado em termos da adoção das nTICs, mas tal não se comprova nesse estudo.

O papel dos atributos de influências interpessoais é mínimo, mesmo quando se flexibiliza as exigências de rejeição de Ho. È preciso descer ao nível de significância de 0,20 para se ver a associação das variáveis 'Habilidade para executar tarefas relacionadas à EaD '(6,517); 'Grau em que as preferências e ações do respondente influenciam as decisões do grupo e das pessoas com que possui interações sociais' $(6,069)$, 'Grau em que as ações do respondente influenciam na promoção de sua imagem perante os outros' $(6,251)$ e 'Grau em que as ações do respondente influenciam a ação de seus pares' $(6,9)$.

O estudo revela também que algumas variáveis independentes (Quadro 1) não apresentaram associações com nenhuma das variáveis dependentes em estudo. 


\begin{tabular}{|c|l|}
\hline ATRIBUTOS & \multicolumn{1}{c|}{ VARIÁVEIS } \\
\hline $\begin{array}{c}\text { Característica Pessoal - } \\
\text { Conhecimento }\end{array}$ & Conhecimento prévio tarefas relacionadas à EaD. \\
\hline $\begin{array}{c}\text { Características Percebidas - } \\
\text { experimentabilidade }\end{array}$ & $\begin{array}{l}\text { Grau com respondente pode descobrir como as nTICS funcionam e } \\
\text { dissipar incertezas a seu respeito. }\end{array}$ \\
\hline $\begin{array}{c}\text { Influências interpessoais } \\
\text { Utilitárias }\end{array}$ & $\begin{array}{l}\text { Grau em que preferências e ações do respondente influenciam as } \\
\text { decisões do grupo e das pessoas com quem possui interações sociais. }\end{array}$ \\
\hline $\begin{array}{c}\text { Influencias interpessoais de } \\
\text { valor }\end{array}$ & $\begin{array}{l}\text { Grau em que o uso das nTICs ajudará na promoção da imagem do } \\
\text { respondente perante seus pares. }\end{array}$ \\
\cline { 2 - 2 } & $\begin{array}{l}\text { Grau em que ações do respondente influenciam na promoção de sua } \\
\text { imagem perante os outros. }\end{array}$ \\
\hline
\end{tabular}

Quadro 1 Variáveis para as quais não se pode rejeitar H0.

Fonte: dados da pesquisa

As variáveis do Quadro 1 apresentaram surpreendente resultado de que o conhecimento prévio de tarefas relacionadas à $\mathrm{EaD}$ não venha a contribuir para a propensão de adoção de nTics, já que o projeto tem o objetivo último de promover a $\mathrm{EaD}$ nas instituições. Assim como também surpreende o fato de a percepção da facilidade de funcionamento da inovação não ter apresentado grau de associação com as variáveis dependentes, já que esta é uma variável independente clássica nos estudos de adoção de novas tecnologias e tem sempre sido reportada favoravelmente. Por fim, três dos nove atributos relativos às influências interpessoais também não se associam com nenhuma das variáveis dependentes. Nesse estudo exploratório não se tem ainda respostas para tal comportamento.

$\mathrm{Na}$ tabela a seguir apresentam-se as variáveis para as quais pode-se rejeitar hipótese nula de que não há associação entre os grupos.

\begin{tabular}{|l|c|c|}
\hline \multicolumn{2}{|c|}{ CARIÁVEIS INDEPENDENTES } & \multicolumn{1}{c|}{ VAR. DEPENDENTES } \\
\cline { 2 - 3 } & EXP & INT \\
\hline \multicolumn{2}{|c|}{ CARTERÍSTICAS PESSOAIS } & 13,118 \\
\hline Sua experiência com produtos/assuntos relacionados ou à NTICs. & - & 10,541 \\
\hline Sua experiência com produtos/assuntos relacionados ou à EAD. & - & 10,912 \\
\hline Sua familiaridade com as nTICs. & 10,507 & $\mathbf{3 1 , 9 7 2}$ \\
\hline Sua familiaridade com a EAD. & 10,355 & 13,892 \\
\hline Sua Habilidade para executar tarefas relacionadas às NTICs. & - & $\mathbf{2 9 , 2 0 8}$ \\
\hline Sua Habilidade para executar tarefas relacionadas às EaD. & - & $\mathbf{2 6 , 5 5 8}$ \\
\hline Seu Conhecimento prévio tarefas relacionado às nTICs. & - & - \\
\hline $\begin{array}{l}\text { Característica inovadora: Grau de esforço que está disposto(a) a realizar para } \\
\text { adquirir uma inovação assim que ela é lançada. }\end{array}$ & 10,379 & \\
\hline \multicolumn{2}{|c|}{ CARACTERÍSTICAS PERCEBIDAS } & - \\
\hline $\begin{array}{l}\text { Vantagem relativas das nTICs sobre métodos atuais na produção de } \\
\text { conteúdo educacional. }\end{array}$ & 9,972 \\
\hline
\end{tabular}




\begin{tabular}{|l|c|c|}
\hline $\begin{array}{l}\text { Grau de compatibilidade das nTICs com os valores existentes em seu meio } \\
\text { em relação à produção de conteúdo educacional. }\end{array}$ & $\mathbf{1 2 , 1 1 1}$ & - \\
\hline $\begin{array}{l}\text { Grau de compatibilidade das nTICs com suas necessidades relativas à } \\
\text { produção de conteúdo educacional. }\end{array}$ & - & 11,041 \\
\hline $\begin{array}{l}\text { Complexidade: Grau de dificuldade de compreensão e uso das nTICs na } \\
\text { produção de conteúdo educacional. }\end{array}$ & - & 11,773 \\
\hline \multicolumn{2}{|c|}{ INFLUÊNCIAS INTERPESSOAIS INFORMATIVAS } \\
\hline $\begin{array}{l}\text { Grau em que o respondente busca informações com pessoas que trabalham } \\
\text { em sua profissão. }\end{array}$ & 11,297 & 11,046 \\
\hline $\begin{array}{l}\text { Grau em que os pares procuram informações com o respondente em sua } \\
\text { profissão. }\end{array}$ & - & \\
\hline \multicolumn{2}{|c|}{ INFLUÊENCIAS INTERPESSOAIS DE VALOR INTERPESSOAIS UTILITÁRIAS } \\
\hline $\begin{array}{l}\text { Grau em que a decisão do respondente é influenciada pelas preferências e } \\
\text { ações do grupo e das pessoas com que possui interações sociais. }\end{array}$ & 11,705 & \\
\hline \multicolumn{2}{|c|}{10,026} & $\mathbf{1 5 , 0 5 9}$ \\
\hline Grau em que as ações do respondente influenciam a ação de seus pares. & 105 \\
\hline
\end{tabular}

EXP $=$ grau de experimentação

INT = grau de intenção de continuar/ampliar a utilização das nTICs na produção.

Fonte: dados da pesquisa

Entre as variáveis dependentes do estudo o grau de intenção de continuar/ampliar a utilização das nTICS na produção de materiais didáticos (INT) foi melhor explicada que o grau de experimentação dos conteúdos trabalhados (EXP).

Têm maior força na explicação do grau de intenção (INT) as variáveis da categoria características pessoais, entre elas Familiaridade com a EaD, Habilidade para executar tarefas relacionadas às $\mathrm{EaD}$ e Conhecimento prévio tarefas relacionado às nTICs. As variáveis ou atributos de percepção da tecnologia tiveram um efeito menor na explicação da variável dependente INT. Observa-se que apenas três atributos exercem efeito influenciador, embora pequeno: vantagens relativas, compatibilidade e complexidade. Da mesma forma, a intenção de continuar/ampliar, nesse estudo, foi influenciada por apenas duas variáveis do grupo influências interpessoais, sendo o grau em que os pares procuram informações com o respondente em sua profissão e o grau em que as ações do respondente influenciam a ação de seus pares.

O grau de experimentação dos conteúdos trabalhado (EXP) foi razoavelmente explicado por três variáveis de características pessoais: familiaridade com as nTICs; familiaridade com as $\mathrm{EaD}$ e característica inovadora medida pelo grau de esforço para adquirir uma inovação. $\mathrm{O}$ grau de experimentação é também explicado pelo grau em que o respondente busca informações com pessoas que trabalham em sua profissão (categoria das 
influências interpessoais informativas) e pelo grau em que as ações do respondente influenciam a ação de seus pares (influencias interpessoais de valor).

É relevante mencionar que dos nove atributos pessoais em estudo, apenas um não é útil à explicação da propensão à adotar as nTICs.

\section{CONCLUSÕES E SUGESTÕES}

O estudo aponta algumas influências na propensão à adoção de novas tecnologias por professores na produção de conteúdos educacionais. Esse conceito foi medido por meio dos constructos: EXP - grau de experimentação dos materiais produzidos e INT - grau de intenção de continuar/ampliar a utilização das nTICs na produção de materiais didáticos. Como a suposição desse estudo é de que quanto mais uma tecnologia é utilizada pelo individuo mais ela é adotada por ele, tem maior importância os resultados relativos a INT do que os relativos à EXP.

Nesse sentido, verifica-se que a propensão à adoção de novas tecnologias por professores na produção de conteúdos educacionais nesse estudo foi positivamente influenciada por todas as variáveis dos atributos pessoais, com exceção da variável Característica inovadora (o grau de esforço que o respondente está disposto a realizar para adquirir uma inovação assim que ela é lançada).

Os resultados desse estudo permitem afirmar que propensão à adoção das nTICs na produção de conteúdos didáticos por parte dos professores participantes desse projeto está positivamente associada ao grau de envolvimento desses professores na modalidade de ensino a distancia e no uso de novas tecnologias. Quanto maior a habilidade e a familiaridade dos respondentes em relação a esses dois conceitos, e quanto maior o conhecimento em relação às nTICs, maior é grau de intenção de continuar/ampliar a utilização das nTICs na produção de materiais didáticos (INT).

Entre os atributos de percepção, observa-se que quanto maior a percepção de compatibilidade das nTICs tanto com os valores existentes no meio quanto com as necessidades relativas à produção de conteúdo educacional maior a propensão para adotar as nTICs.

O que se depreende dos resultados do estudo é que para incentivar a propensão dos professores à adoção das nTICs na produção de conteúdo faz-se necessário um trabalho prévio. Esse trabalho, talvez a titulo de envolvimento, deveria incentivar a familiaridade com 
as nTICs e a EaD de modo a favorecer a percepção de suas vantagens e compatibilidades. Além disso, esse trabalho deve ser iniciado com grupos de professores que exerçam papel relevante em suas áreas de atuação, para que com isso se possa contar com o efeito influenciador e motivador, atuando esses como formadores de opinião.

\section{REFERÊNCIAS}

COOPER, R. B.; ZMUD, R. W. Material requirements planning system infusion. Omega, v.17, n.5, p.471. 1989.

DAVIS, F.D. Perceived usefulness, perceived ease of use and user acceptance of information technology. MIS Quarterly, 13, p 319-340, September. 1989.

DAVIS, F.D; BAGOZZI, R P; WARSHAW, P.R. User acceptance of computer technology: a comparison of two theoretical models. Management Science. Vol 35, 8, 982-1003, August, 1989.

FICHMAN, R. G. ; KEMERER, C. F. The illusory diffusion of innovation: An examination ofassimilation gaps. Information Systems Research, v.10, n.3, Sep, p.255-275. 1999.

GATIGNON, H.; ROBERTSON, T. S. Innovative decision processes. In: ROBERTSON, T. S.; KASSARJIAN, H. H. Handbook of consumer behavior. New Jersey: Prentice-Hall, p. 316-348. 1991.

JEYARAJ, A.;ROTTMAN, J. W. ; LACITY, M. C. A review of the predictors, linkages, and biases in IT innovation adoption research. Journal of Information Technology, v.21, n.1, p.1. 2006.

HUFF, S.L.; McNAUGHTON, J. Diffusion of an Information Technology Innovation. Business Quarterly . London vol 56, Num 1, pg 25-31. 1991.

MOORE, G. C. ; BENBASAT, I. Development of an instrument to measure the perceptions of adopting an information technology innovation. Information Systems Research, v.2, n.3, p.192-222.1991.

PÁDUA JÚNIOR, Fábio Pimenta de \& PRADO, Paulo Henrique Muller. A adoção de inovações em produtos de alta tecnologia por jovens: O caso do telefone celular. Revista Eletrônica de Ciência Administrativa (RECADM) Faculdade Cenecista de Campo Largo, v. 5 , n. 1 , maio/2006

PEREZ, G. Adoção de inovações tecnológicas: um estudo sobre o uso de sistemas de informações na área da saúde. Tese de doutorado. Universidade de São Paulo. 2006.

ROGERS, E. M. Diffusion of innovations. 5. ed. New York: Free Press, 2003.

SANTOS, André Moraes dos. Fatores influenciadores da adoção e infusão de inovações em TI. Anais do IV SEGeT - Simpósio de Excelência em Gestão e Tecnologia, da Associação Educacional Dom Bosco. 2007. 
TORI, R. A distância que aproxima. Revista de Educação a Distância, v.1, n.2, p.1-7, 2002. Disponível em: <http://www.abed.org.br>. Acesso em: dez. de 2009.

\section{APÊNDICE - QUESTIONÁRIO}

Avalie suas respostas numa escala de pontos de 1 a 5 sendo:

$1=$ muito pouco/quase nenhum/ruim e $5=$ Muito grande/ totalmente/muito bom

Antes do início dos projetos com as nTICs na CEAD como você avalia

a) Sua experiência com produtos/assuntos relacionados às nTICs.

b) Sua experiência com produtos/assuntos relacionados ou à EAD.

(1) (2) (3) (4) (5)

c) Sua familiaridade com as nTICs.

d) Sua familiaridade com a EAD.

e) Sua Habilidade para executar tarefas relacionadas às NTICs.

(1) (2) (3) (4) (5)

f) Sua Habilidade para executar tarefas relacionadas às EaD.

g) Seu Conhecimento prévio tarefas relacionado às nTICs.

h) Seu Conhecimento prévio tarefas relacionado à EaD.

i) Seu grau de esforço que estou disposto a realizar para adquirir uma inovação assim que ela é lançada.

j) As vantagem relativas das nTICs sobre métodos atuais na produção de conteúdo educacional.

k) Grau de compatibilidade das NTICs com os valores existentes em seu meio em relação à produção de conteúdo educacional.

1) Grau de compatibilidade das NTICs com suas necessidades relativas à produção de conteúdo educacional.

m) Grau de dificuldade de compreensão e uso das NTICs na produção de conteúdo educacional

(1) (2) (3) (4) (5)

(1) (2) (3) (4) (5)

(1) (2) (3) (4) (5)

(1) (2) (3) (4) (5)

(1) (2) (3) (4) (5)

(1) (2) (3) (4) (5)

(1) (2) (3) (4) (5)

n) Grau com que vc pode descobrir como as nTICS funcionam e e dissipar incertezas a seu respeito.

o) Grau em que vc procura informações com pessoas que trabalham em sua profissão

p) Grau em que seus pares procuram informações com você em sua profissão.

(1) (2) (3) (4) (5)

(1) (2) (3) (4) (5)

q) Grau em que sua decisão é influenciada pelas preferências e ações do grupo e das pessoas com que possui interações sociais.

(1) (2) (3) (4) (5)

r) Grau em que suas preferências e ações influenciam as decisões do grupo e das pessoas com que possui interações sociais.

s) Grau em que o uso das nTICs o ajudará na promoção de sua imagem perante seus pares.

t) Grau em que suas ações influenciam na promoção de sua imagem perante os outros.

u) Grau em que suas ações influenciam a ação de seus pares. 